\title{
INDICADORES DE EMPLEABILIDAD: DE LA INCLUSIÓN AL DESARROLLO DE LAS CARRERAS LABORALES
}

Lucía Llinares Insa

Departamento de Psicología Social, Facultat de Psicologia

Universitat de València

\author{
Ana Isabel Córdoba Iñesta \\ Juan José Zacarés González \\ Departamento de Psicología Evolutiva y de la Educación, Facultat de Psicologia \\ Universitat de València
}

Pilar González-Navarro

IDOCAL, Universitat de València

\section{RESUMEN}

De la misma forma que la educación tiene la responsabilidad de fomentar la inserción laboral, la empleabilidad está reconocida como una competencia para el logro y mantenimiento del empleo, es por ello que, la empleabilidad es un eje central en la educación. En la Declaración de Incheon y el Objetivo 4 de Educación 2030 de Desarrollo Sostenible se destaca la importancia de las competencias básicas para hacer frente a la vulnerabilidad social y con el fin de conseguir el pleno empleo. Sin embargo, en la literatura científica sobre empleabilidad no hay acuerdo sobre los indicadores que la integran. Nuestro objetivo es delimitar qué indicadores resultan relevantes para el desarrollo de la empleabilidad. Para ello, analizamos los indicadores de empleabilidad que utilizan 30 empresas de inserción (EI) españolas para la realización de los itinerarios formativos de los trabajadores de inserción (PTI). Los resultados muestran una gran heterogeneidad de indicadores. La mayoría de ellos son características individuales, en menor medida hemos encontrado circunstancias personales y prácticamente inexistentes aparecen factores contextuales. Este artículo finaliza resaltando el vínculo formación-trabajo y la formación integral de la persona.

PALABRAS CLAVE: Empleabilidad, educación, integración social, formación para el empleo.

CLAVES ECONLIT: H53, J6, J21.

Cómo citar este artículo/How to cite this article: LLINARES INSA, L., CÓRDOBA IÑESTA, A.I., ZACARÉS GONZÁLEZ, J.J. \& GONZÁLEZ-NAVARRO, P.: “Indicadores de empleabilidad: de la inclusión al desarrollo de las carreras laborales", CIRIEC-España, Revista Jurídica de Economía Social y Cooperativa, n³ 36, 2020, pp. 365-387. DOI: 10.7203/CIRIEC-JUR.36.17016. 
(pp. 365-387)

\section{EMPLOYABILITY INDICATORS: FROM INCLUSION TO CAREER DEVELOPMENT}

\section{EXPANDED ABSTRACT}

The Incheon Declaration and the Objective 4 of Education 2030 of Sustainable Development (UNESCO, 2015) highlight the relevance of basic competences to face social vulnerability promoting full employment. Nevertheless, in the scientific literature on employability there is not agreement about the employability' indicators.

However, at present two of the challenges facing most countries are the fight against social exclusion and the creation of inclusive education. The Lisbon European Council emphasizes the need for education and training in Europe to generate a knowledge society and adapt to the needs of the world of work (RLCEur $2005 \backslash 1178$, of May 24, 2005; Declaration of the Sorbonne, 1998). The challenge has been translated into a new community educational approach: skills for employment (García and Ibáñez, 2006).

Nevertheless, the notion of employability is used in many contexts and it has multiple and diverse meanings (McQuaid and Lindsay, 2005) and the lack of systematization makes it a diffuse concept (De Grip, Van Loo, and Sanders, 2004). Thus, we need to specify the employability indicators that allow identifying the skills to be trained, the learning contents, and the elements to be evaluated, especially in vulnerable groups.

Employability has been approached from two clearly differentiated perspectives (Llinares, Zacarés, and Córdoba, 2016). According to the individualistic perspective employability is an individual characteristic that must be promoted for the achievement and maintenance of the job (Finn, 2000; Formichella, and London, 2005; McQuaid and Lyndsay, 2005). However, there is no agreement on the most relevant individual characteristics to promote (Llinares et al., 2016).

Second, the critical perspective affirms that the people' qualification constitutes a social construction that is based on class hierarchies and contributes to the production and legitimation of differentiation and social exclusion. From this perspective, the idea of employability is promoted as a broader concept where individual factors, personal circumstances, and contextual factors must be considered (McQuaid and Lindsay, 2005; Llinares et al., 2016).

However, there is not clarity about employability indicators, especially in vulnerable groups. An example is provided by the Worker Insertion Social Enterprises (WISE) (FAEDEI-AERES, 2014), companies that prepare vulnerable people to access to ordinary employment in Spain (L44 / 2007, of December 13, RCL 2007 \2249). 
The WISEs use evaluation tools to collect information from the insertion worker to improve its employability. We analyzed The Instrument for the Analysis of Employability Indicators (IPAIE) (Llinares, Córdoba, and Zacarés, 2011) to analyze these tools. Nevertheless, in different previous investigations (e.g. Córdoba, Llinares, and Zacarés, 2013; Llinares, Córdoba, and Zacarés, 2012) it is observed that, although it includes a large number of indicators, it is a limited instrument to collect the wide variety of relevant employability indicators.

Our goal is to delimitate which dimensions are relevant to the employability' development. Therefore, we analyze the employability' indicators used by Spanish Work Integration Social Enterprises (WISE) to design the training itineraries of the insertion workers.

In order to define the indicators to measure employability, we analyzed the assessment instruments used by WISE. We collected all instruments used by 30 WISE, with a total of 220 instruments. We used the IPAIE that compiles the indicators into three broad categories (McQuaid and Lindsay, 2005): 1) Individual factors, referred to the worker. 2) Personal circumstances, referred to family responsibilities, access to resources. 3) External factors: determining factors of the socioeconomic environment related to the labor market (Córdoba et al., 2013).

In order to identify the employability indicators for vulnerable people, and specifically in WISE, we classified the elements that evaluated employability according the IPAIE. Seven specialists linked to the area of social exclusion carried out the categorization and three of them analyzed the agreement index. Subsequently, we analyzed the presence or absence of the elements in each category. When the elements of the employability did not apply to any category we placed it in the '0ther' section in order to detect those specific parameters of the vulnerable groups. We analyzed it using frequencies and percentages.

Results showed a great heterogeneity of indicators, although they were mainly focused on individual dimensions (skills and personal attributes) (89.70\%); very rarely on personal circumstances (access to resources, family responsibilities) $(9.66 \%)$; and very few contextual factors $(0.64 \%)$.

Analyzing the open category of '0thers', employability in vulnerable groups is conceived as a competence and / or ability of the person that includes personal attributes and minimally influenced by the personal circumstances. The most important were personal care, interpersonal communication, or specific professional skills. Regarding the content that reflects the deficiencies of pre-employment socialization, we found absenteeism, job search techniques and training / insertion itineraries. 
This research ends with a debate-reflection about the training-work ling and about the integral formation of the person.

We found that evaluation of employability from WISE is mainly based on personal competencies as personal skills and attributes, although they analyse some variables that can mediate it. Thus, it emphasizes the person's responsibility to develop their work (Fugate, Kinicki, and Ashforth, 2004; Salognon, 2007), which de-responsibilizes the rest of the social agents (De Lara and Andrade, 2008). From this perspective, inequalities are due to the person's lack of ability to apply for certain jobs. This individual perspective is a legitimizing element of the social and labor context.

However, not all the elements that allow the person to access and maintain employment depend directly on the worker, since having employability skills does not guarantee obtaining and keeping a job. Therefore, on another hand, from a critical perspective employability does not depend solely on the responsibility of the individual, but also on the needs of the market, the preferences of the employer, the characteristics of the job or the worker personal circumstances (Bauman, 2005; Beck and Beck-Genshein, 2002). Moreover, employability is a complex concept, which must include macro (social, political and ideological structures) and micro aspects (individual factors and immediate social contexts) to cope with unemployment and marginalization (Llinares et al., 2016).

In this sense, the data derived from the analysis of instruments reflect that in vulnerable groups we have to broaden the vision of the usual employability indicators to both individual and personal circumstances that clearly affect the process of social and labor insertion of individual at risk.

To conclude, there is a need to generate new empowering educational projects with the individual that seek lifelong training and empowerment. This requires the analysis and intervention on individual employability factors, and a more realistic and global perspective that considers the specific limiting circumstances starting from Bronfenbrenner's bioecological model (Bronfenbrenner and Morris, 1998, 2006), that integrates the individual in their context taking into account their personal circumstances and contextual factors.

On the other hand, we have to continue deepening on the elements of employability to be fostered in the educational system that provide the subject with comprehensive training, including the acquisition of knowledge and professionalization as claimed in the current context. In this way, employability becomes the instrument of analysis and intervention, but also of reflection.

KEYWORDS: Employability, education, social integration, job training. 


\section{SUMARI0 ${ }^{1}$}

1. Introducción. 2. Método. 3. Resultados: el núcleo central de los indicadores de la empleabilidad. 4. Discusión. Referencias bibliográficas.

\section{Introducción}

Los cambios en el mundo laboral generados por la globalización y las nuevas tecnologías han modificado el panorama de la seguridad en el empleo. El neoliberalismo ha generado el paso de una carrera profesional tradicional (donde los empleados leales trabajan en una única empresa y prosperan en ella) a una perspectiva de carrera sin seguridad en la empresa (Bernstrom, Drange y Mamelund, 2019). Esto hace que el término 'empleabilidad' se convierta en una alternativa a la seguridad en el empleo, se relacione con el desarrollo de carrera laboral y se aplique a toda la población en edad laboral. En este sentido, en los últimos años se ha incrementado la complejidad del concepto de empleabilidad y su multidimensionalidad (Williams, Dodd, Steele y Randall, 2016). Se han desarrollado multitud de intervenciones para su fomento (ver, por ejemplo, Hazelzet, Picco, Houkes, Bosma y de Rijk, 2019). Sin embargo, no hay un acuerdo en los elementos que la componen (Gabor, Blaga y Mastis, 2019). Es por ello que en esta investigación se pretende delimitar los elementos que forman parte de la empleabilidad.

El término de empleabilidad se utilizó tradicionalmente para diferenciar a los aptos de los no aptos para el logro y mantenimiento del empleo (Rentería y Malvezzi, 2008). Posteriormente, comenzaron a desarrollarse y coexistir en la literatura corrientes teóricas diferentes (Nauta, van Vianen, van der Heijden, van Dam y Willemsen, 2009). De modo general, la empleabilidad se ha abordado desde dos perspectivas claramente diferenciadas (Llinares, Zacarés y Córdoba, 2016): la perspectiva individualista y la perspectiva crítica.

La perspectiva individualista entiende la empleabilidad como característica individual para la consecución y mantenimiento del puesto de trabajo (Finn, 2000; Formichella y London, 2005; McQuaid y Lyndsay, 2005). Esta perspectiva permite clasificar a la población activa entre la que es empleable y la que no lo es (Fugate, Ki-

1. Este trabajo se deriva del proyecto que lleva por nombre 'EMPLEAM: Evalúa y mejora la empleabildiad' concedido en la 'Convocatòria d'Ajudes per a la Realització de Projectes de Prova de Concepte 209. Programa Valoritza i Transfereix’ del Vicerectorat d'Innovació i Transferència de la Universitat de València. 
nicki y Ashforth, 2004; Moreland, 2006; Thijssen, Van der Heijden y Roco, 2008). Sin embargo, no existe acuerdo sobre las características individuales más relevantes a promover (Llinares et al., 2016). La mayoría de los estudios se limitan al análisis del nivel educativo, donde el grado de escolarización es utilizado como condición para la incorporación de un individuo a un puesto de trabajo, actuando como filtro (Formichella y London, 2005). Otros autores, sin embargo, destacan aspectos tangibles e intangibles como la capacidad de comunicación, el manejo de procesos de organización, trabajo en equipo (Brunner, 2000) y el aprender a aprender (Rentería y Malvezzi, 2008). En este sentido, Fugate y Kinicki (2008) consideran a la empleabilidad como un conjunto de diferencias individuales que predisponen al individuo a la adaptabilidad proactiva específica para el empleo. Esta perspectiva centrada en las diferencias individuales en el desarrollo de la carrera laboral es la perspectiva más desarrollada y mayoritaria en los discursos científicos y sociales (Hogan et al, 2013).

En segundo lugar, desde una perspectiva crítica se percibe la empleabilidad como eje articulador de la vida social (Lindsay y Serrano, 2009). Se parte de la concepción de que la cualificación o competencia de las personas constituye una construcción social que, por una parte, se basa en las jerarquías de clase y, por otra, contribuye a la producción y legitimación de la diferenciación y exclusión social. Así, formar para la empleabilidad implica educar a los individuos para que 'aprendan a aprender' de manera permanente, es decir, a el hacer (habilidades técnicas) y a el ser (habilidades personales). En el mundo formativo, por tanto, afecta a los resultados del aprendizaje como eje central que, a su vez, tienen que poder demostrarse en el marco productivo. Se analiza así el ajuste de competencias a partir de la relación entre el currículo educativo (objetivos, contenidos, metodología y evaluación), los perfiles profesionales requeridos por la sociedad y la lucha contra la exclusión social. De esta forma, desde el sistema educativo se prepara al futuro trabajador para garantizar una trayectoria laboral lo más exitosa y satisfactoria posible (García y Ibánez, 2006). La eficacia-eficiencia, rentabilidad y calidad de la educación se visualizará en el desarrollo de las áreas educativas que demanda el mercado y en el entrenamiento de los recursos humanos. Desde esta perspectiva crítica se fomenta la idea de la empleabilidad como un concepto más amplio donde se deben considerar tanto los factores individuales como las circunstancias personales y factores contextuales (McQuaid y Lindsay, 2005; Llinares et al., 2016). Así pues, cada perspectiva teórica tiene una aproximación diferente a la realidad laboral y pone de relieve indicadores y dimensiones distintas. Es más, su vinculación con las carreras laborales supone la incorporación de nuevos componentes dentro de las mismas perspectivas y, como consecuencia, se evidencia una falta de acuerdo en ellos (Williams et al, 2016). 
Actualmente, se han realizado numerosos estudios para delimitar los elementos de la empleabilidad (ver, por ejemplo, Mohd, Mustapha, Malik, Mohamad, Rasul y Mokhtar, 2013; Chacón-Sánchez, Casas-Mateus, Alvarado, 2020). Sin embargo, aunque no existe acuerdo sobre los indicadores que la componen, parece que hay consenso en la consideración de que éstos son características o atributos personales vinculados con el trabajo y que son apreciados por los empresarios (Fahimirad, Nair, Kotamjani, Mahdinezhad y Feng, 2019). En este sentido, la empleabilidad es un área de intervención socio-educativa que debe responder a los cambios del mundo del trabajo por medio de nuevas trayectorias en la carrera laboral (Olson y Shultz, 2013). Así, el apoyo profesional en el desarrollo de la carrera laboral se está convirtiendo en un área de trabajo de algunos agentes sociales vinculados a la educación y a la acción social. Su objetivo es formar y desarrollar destrezas y actitudes para que la persona planifique y programe sus experiencias de aprendizaje y tome decisiones sobre su participación en la vida laboral (Terzaroli y Oyekunle, 2019).

Cualquier nueva situación laboral requiere la planificación de carreras laborales y redefinir destrezas, habilidades, talentos, conocimientos, etc. de las personas que acceden y que están en el mundo laboral. Es más, hay consenso en que la principal razón del desempleo en todos los estamentos sociales y formativos residen en la falta de empleabilidad (ver, por ejemplo, Ang, 2015 para el análisis de los estudiantes universitarios; ver, por ejemplo, Ebuenyi, Guxens, Ombati, Joske, Bunders-Aelen y Regeer, 2019 para las personas con diversidad funcional; ver, por ejemplo, Storme, De Lannoy, Leibbrant, De Boeck y Mudiriza, 2019 vulnerability youngs). Hay autores que también han encontrado la relación entre la empleabilidad y el éxito en la carrera (ver por ejemplo, Olson y Shultz, 2013) y los nuevos modelos de carrera laboral y la empleabilidad como garante del empleo y sustitutoria de la seguridad (Bernstrøm, Drange y Mamelund, 2019). Así pues, la empleabilidad es una herramienta compensatoria para los problemas estructurales vinculados al empleo y/o potenciadora del éxito profesional. En este contexto, la educación formal, informal y no formal tiene como misión generar conocimiento y capacidad de adaptación a las condiciones laborales para el fomento de la empleabilidad. De hecho, en la Declaración de Incheon y el Objetivo 4 de Educación 2030 de Desarrollo Sostenible (UNESCO, 2015) se destaca la importancia del aprendizaje de las competencias básicas para potenciar el acceso al empleo. Así, en la actualidad, la mayoría de países tienen que afrontar la lucha por el empleo y la integración social por medio del fomento de la empleabilidad (Declaración de Incheon y Marco de Acción ODS 4-Educación 2030, UNESCO, 2015).

En este contexto, la educación formal debe establecer la relación entre educación y empleabilidad. Su misión es generar conocimientos y capacidad de adaptación a las 
condiciones laborales, incluir contenidos de la socialización pre-laboral (Formichella y London, 2005) y, en definitiva, fomentar la competencia de la empleabilidad (Campos, 2003). En concreto, la educación superior es considerada un campo de acción social que está condicionado por las características contextuales y por sus demandas sociales y económicas (Ang, 2015). En ella, la empleabilidad es contenido, herramienta e indicador de calidad (Orellana, 2018). Por su parte, la educación no formal cumple una función en la inserción de los individuos menos cualificados en el mercado laboral (desde las ONGs y la comunidad, en general, hasta las organizaciones de empresarios y trabajadores). Además, debe promover el empoderamiento de las personas y la participación ciudadana, fomentar la creación de nuevas organizaciones y proporcionar apoyo económico y financiación para el autoempleo (Formichella y London, 2005). Esto supone la consideración de nuevas alianzas entre educación/formación, ámbito social y empleo y nuevos indicadores en materia de educación y formación donde la empleabilidad parece ser el elemento central (Pérez, 2003; Ventura, 2005). Esto plantea nuevos retos políticos, sociales y educativos a toda la sociedad y requiere una clarificación de los elementos que están en su base. Sin embargo, no existe consenso sobre los elementos que la configuran.

En la actualidad, nos encontramos en una situación laboral que se caracteriza por una nueva crisis que han supuesto el fin del pleno empleo (Bernad y Martínez, 2011), el surgimiento de nuevos modelos de carreras laborales (De Cuyper, Mauno, Kinnunen y Makikangas, 2011), la precarización del empleo (Recio y Banyuls, 2004) y la disolución de las diferenciaciones entre puestos de trabajo (Amigot y Martínez, 2013). Forrier y Sels (2003) afirman que la empleabilidad, en este contexto, es el mecanismo estrella de protección del trabajador. Esto hace que pase de ser percibida como un elemento explicativo del desempleo de determinados grupos vulnerables a ampliarse a toda la población. Este nuevo contexto le dota de múltiples y diversos significados (Bernstrøm et al, 2019) con diferentes antecedentes y consecuentes (Drange, Bernstrøm y Mamelund, 2018) y la convierten en un concepto difuso (De Grip, Van Loo y Sanders, 2004) aunque ampliamente utilizado. Además, en el mundo del empleo y en el contexto educativo no se han consensuado cuáles son los indicadores de empleabilidad (Gabor, Blaga y Mastis, 2019). Es más, la mayor parte de los estudios presentan estos indicadores utilizando como muestra de estudio a graduados universitarios (ver, por ejemplo, Chacón-Sánchez, Casas-Mateus y Nieto, 2020), trabajadores en activo (ver por ejemplo, Stoffers, Van der Heijden y Jacobs, 2020) o jóvenes vulnerables (ver por ejemplo McDonald, Grant-Smith, Moore y Marston, 2020). Junto a ello, las clasificaciones anteriores restringen los indicadores a las cualidades y las habilidades del individuo (Alpek y Tésits, 2019) de colectivos concretos, lo que no permiten su generalización al conjunto de la población. Es por 
ello que se hace necesario identificar y analizar de forma integral y objetiva cuáles son los indicadores que definen la empleabilidad. En este estudio vamos a realizar este análisis de forma minuciosa y objetiva usando como marco de referencia una perspectiva bioecológica de la empleabilidad (Llinares et al, 2016).

Nuestro estudio hace un número de contribuciones a la literatura. Primero, extendemos los indicadores a las circunstancias personales y los factores contextuales; así pues, el objetivo es generar un listado de indicadores que operacionalicen la empleabilidad desde una perspectiva bioecológica. Además, nosotros extendemos los indicadores a toda la población, generando un listado único para la población general. Esto permitirá una medida similar de las competencias que la componen en todas las personas. Segundo, este estudio puede ser útil a los gabinetes de recursos humanos, a los docentes de primaria, secundaria y formación profesional, a los docentes de formación continua, a los profesores universitarios, a los orientadores, a los agentes sociales vinculados al empleo, etc. ya que les permitirán identificar las competencias a formar, los contenidos de aprendizaje a transmitir y los elementos a evaluar, especialmente en los colectivos considerados en riesgo de vulnerabilidad social por sus características particulares.

\section{Método}

Para la delimitación de los indicadores centrales de la empleabilidad que resultan básicos en el acceso y mantenimiento en el empleo, hemos optado por centrarnos en aquellos que se fomentan en la socialización anticipatoria y que muestran la brecha entre personas empleables y el trabajador en el mercado laboral ordinario. Las empresas de inserción (EI) son las entidades que consiguen altas tasas de incorporación al empleo. Autores como Martínez-Rueda, Galarreta y Aróstegui (2018) ponen de manifiesto la efectividad de las EI en el desarrollo de la empleabilidad de personas con necesidades de inserción social. Las EI son empresas de tránsito al empleo ordinario en España (L44/2007, de 13 diciembre RCL 2007\2249). Estas, dan empleo a personas en riesgo de exclusión social durante un periodo de tiempo delimitado, entre dos y tres años, centrándose en su formación socio-laboral a lo largo del proceso de inserción que conlleva tres fases (FAEDEI-AERES, 2014; Marhuenda-Fluixá, 2018): evaluación inicial o acogida (momento de ingreso del trabajador en la empresa), seguimiento (del proceso que sigue cada trabajador) y tránsito al mercado laboral (momento en que se analiza la adecuación final del trabajador a la empresa ordinaria). Lo esperable es que, en la primera fase, se establezcan como objetivos de intervención los indicadores de empleabilidad de los que adolezca el trabajador y que se evalúe su 
consecución en la fase final, de transición al mercado laboral. Las EIs utilizan una serie de recursos a modo de herramientas de evaluación para recoger información del trabajador de inserción a lo largo del proceso para la mejora de su empleabilidad.

Para la estudiar los indicadores que miden la empleabilidad en cualquier tipo de colectivo se han analizado los instrumentos de evaluación que utilizan las EI para los itinerarios de inserción de los trabajadores de inserción (PTI). Estos itinerarios tienen una duración máxima de tres ańos y son redactados por los trabajadores acompañantes (PTA) conjuntamente con PTI. Los PTA son los agentes sociales que establecen qué se evalúa, qué aspectos resultan deficitarios para la inclusión laboral y cómo formarlo. Los PTA, como agentes sociales vinculados al mundo laboral normalizado y a las dificultades que suelen presentar los PTI para su inclusión, generan instrumentos de evaluación. En esta investigación se han analizado los indicadores que evalúan las EI españolas en su conjunto. Para ello, en colaboración con la Federación Española de Empresas de Inserción (FAEDEI), se solicitaron dichos instrumentos a todas las EI del ámbito nacional. La muestra estuvo compuesta por 220 instrumentos de evaluación de la empleabilidad que utilizaron las EI para su medida.

Para el análisis de los indicadores se amplió el Instrumento para la Medida de la Empleabilidad (IPAIE) de Llinares et a. (2011). Este instrumento recopila y clasifica los indicadores de empleabilidad que se proponen desde la literatura científica y que son aplicables a toda la población y todo tipo de sectores ocupacionales. Éstos se agrupan, siguiendo a McQuaid y Lindsay (2005), en tres grandes categorías que a su vez se dividen en una serie de subcategorías principales (ver figura 1): 1) Factores individuales, que son los que suelen tomarse en consideración en la mayoría de los casos, casi exclusivamente, como los únicos determinantes de la empleabilidad. Se refieren al propio trabajador, como atributos personales (honestidad, disposición para trabajar, motivación intrínseca, actitudes hacia el trabajo); habilidades y competencias (habilidades básicas, adaptabilidad, hábitos laborales o habilidades sociales como trabajo en equipo o gestión positiva del conflicto); salud o movilidad geográfica y flexibilidad laboral. 2) Circunstancias personales, que condicionan el desempeño del trabajo. Se refieren a responsabilidades familiares, cultura del trabajo, acceso a los recursos como transporte, vivienda, apoyo social. 3) Factores externos al sujeto, de los que depende no sólo conseguir un empleo sino también mantenerlo. Se refieren a condicionantes del entorno socioeconómico relacionados con el mercado de trabajo, factores macroeconómicos o políticas de empleo (Córdoba et al., 2013). Junto a ello, el instrumento solicita información sobre su presencia, el grado de operativización y el grado de estructuración. 


\section{Figura 1.}

Los indicadores de la empleabilidad valorados en el IPAIE (Llinares, Córdoba y Zacarés, 2011)

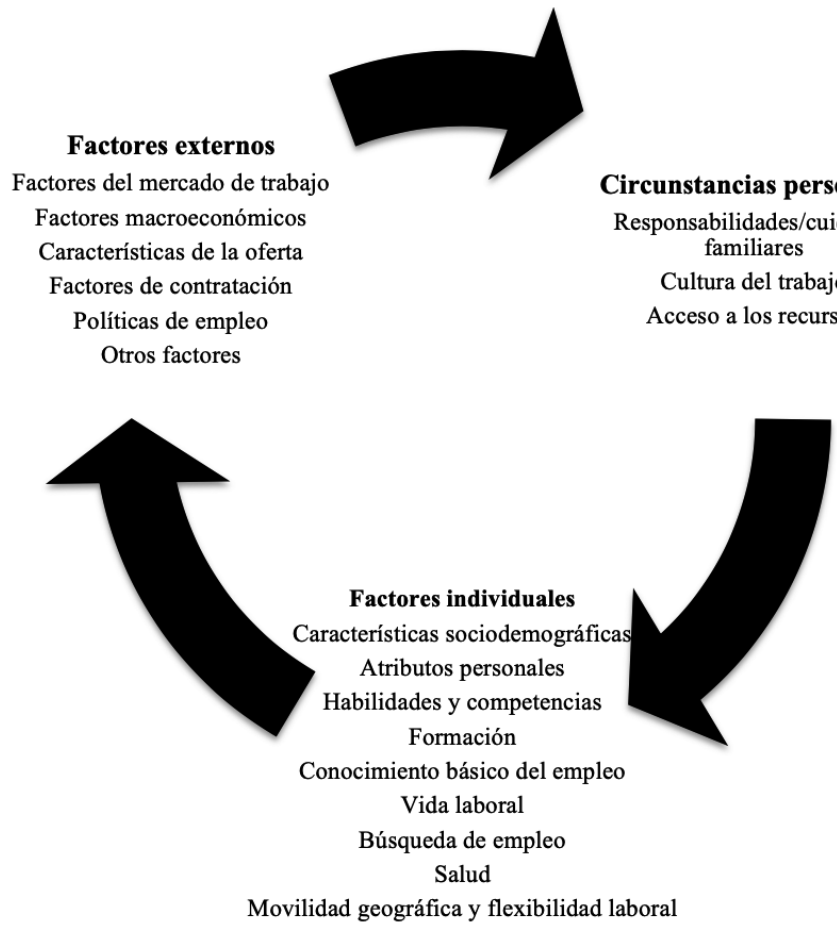

El análisis de los indicadores de la empleabilidad se llevó a través de un análisis de contenido. En primer lugar, se mejoró la categorización del IPAIE por medio de la incorporación de los ítems que aparecían en un apartado de información complementaria que se encontraba en cada subcategoría. Para ello, se tuvieron en cuenta todos los criterios de validez y fiabilidad del análisis de contenido y de la categorización. Posteriormente, se llevó a cabo la codificación del material. Para ello, se seleccionaron siete jueces vinculados al área de Educación, Ciencias Sociales y Salud (dos Doctores en Didáctica y Teoría de la Educación, tres Doctores en Psicología social y dos Doctores en Psicología Evolutiva y de la Educación). La fiabilidad, siguiendo a Porta y Silva (2003), fue calculada con el porcentaje de veces que los codificadores coinciden cuando analizan el mismo documento. En esta investigación el material fue codificado dos veces por dos jueces de diferentes áreas de conocimiento. 


\section{Resultados: el núcleo central de los indicadores de la empleabilidad}

En primer lugar, se analizaron los ítems que no estaban representados en las categorías generales del IPAIE. El objetivo era detectar aquellos elementos específicos de los colectivos vulnerables que les dificultan el acceso al empleo. Los resultados obtenidos de los factores individuales se presentan en las figuras 4 y 5 .

\section{Figura 4.}

\section{Otros indicadores de empleabilidad especificos en colectivos vulnerables}

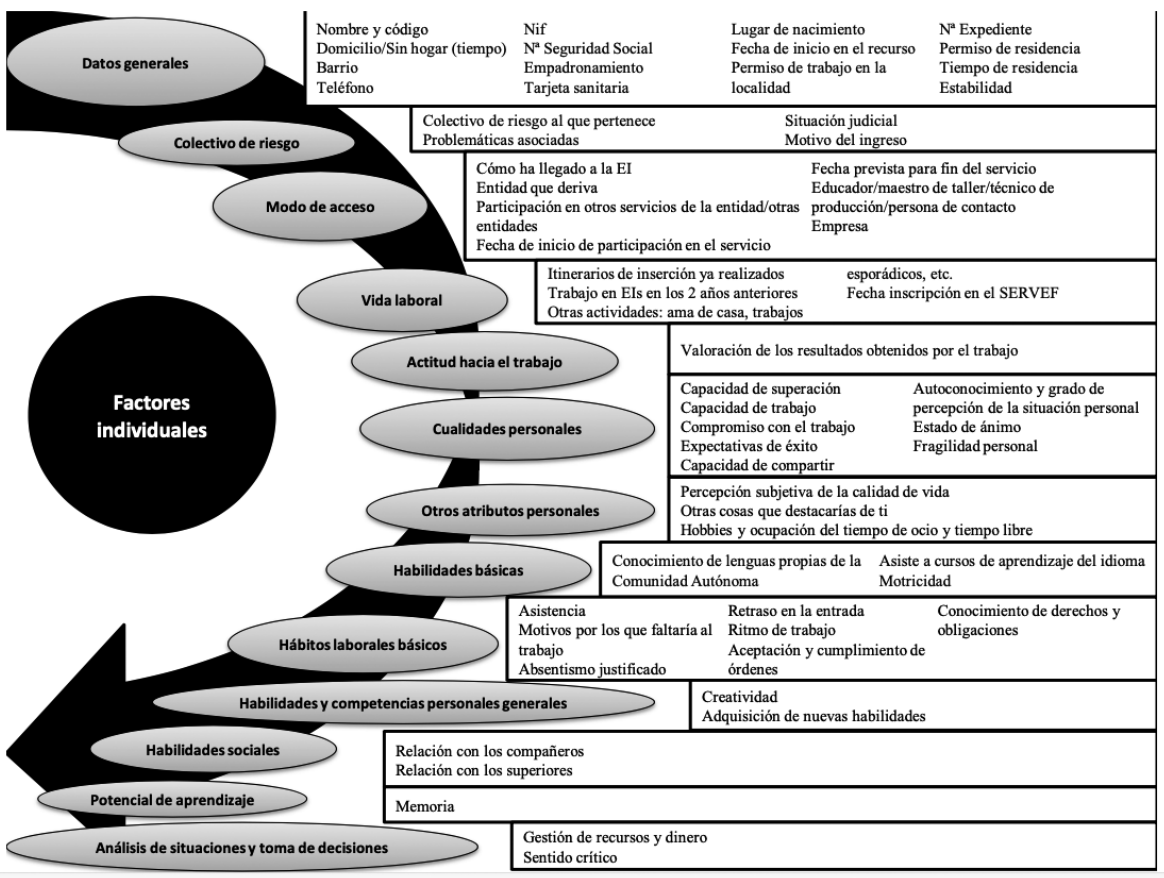

Como puede verse en la figura 4, las características socio-demográficas que no estaban incluidas en el IPAIE se han estructurado en tres subcategorías en función de la información que solicitaban: a) datos generales de la persona, sobre todo en lo que se refiere a su situación legal-administrativa; b) colectivo de riesgo, agrupa a todos los indicadores que preguntan por el tipo de problema que le hace ser considerado población en riesgo de vulnerabilidad; c) modo de acceso, es la subcategoría que pregunta por las vías de acceso a la empresa de inserción y sintetiza la trayectoria de la persona 
por los itinerarios de formación de diferentes organizaciones. Este apartado se completa cuando se pregunta por la vida laboral.

En segundo lugar, en el apartado de atributos personales se resalta la importancia otorgada a la valoración del trabajo bien hecho y a la mejora del mismo, la autopercepción de la capacidad de trabajo y la calidad de vida y se le pide una autorreflexión sobre él mismo y sus preferencias. En tercer lugar, se puede observar la gran importancia otorgada al absentismo/asistencia, cantidad de trabajo realizado y modos de relación social con los compañeros y superiores en la categoría de las habilidades y competencias. Resulta relevante mencionar aspectos como gestión del tiempo y dinero, memoria y motricidad.

Figura 5.

Otros indicadores de empleabilidad especificos en colectivos vulnerables (continuación)

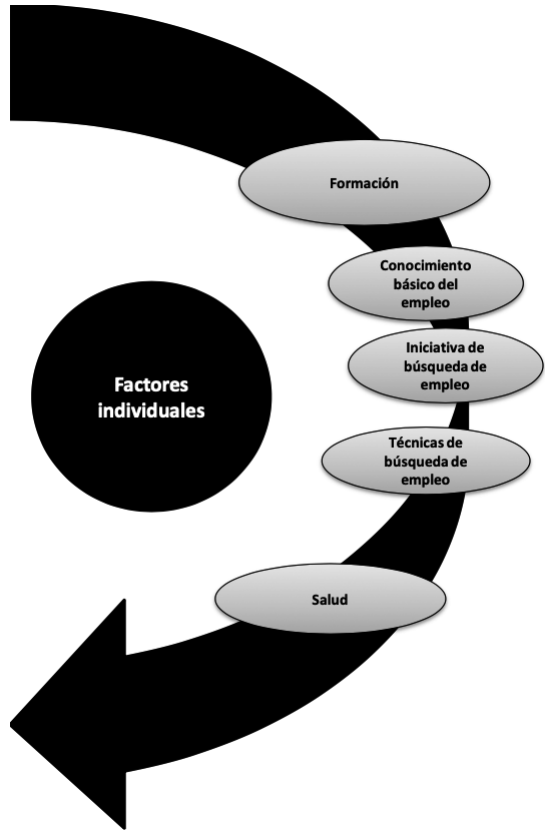

\begin{tabular}{|l|}
$\begin{array}{l}\text { Formación reglada inacabada } \\
\text { Formación complementaria inacabada } \\
\text { Formación en curso } \\
\text { Solicitud de formación } \\
\text { Programas de apoyo escolar }\end{array}$ \\
\hline $\begin{array}{l}\text { Tareas a mejorar } \\
\text { Grado de profesionalidad }\end{array}$ \\
\hline Interés en el autoempleo \\
\hline $\begin{array}{l}\text { Sector en el que se centra la búsqueda de empleo } \\
\text { Dificultades en la búsqueda de empleo } \\
\text { Temores/seguridad en la búsqueda de empleo } \\
\text { Factores de éxito/fracaso en la búsqueda de empleo } \\
\text { Limitación horaria para la búsqueda de empleo } \\
\text { Autopercepción de eficacia en la búsqueda de empleo } \\
\text { Conocimiento de diferentes recursos/programas concretos de orientación } \\
\text { y búsqueda de empleo } \\
\text { Disposición a participar en la búsqueda de empleo }\end{array}$ \\
\hline $\begin{array}{l}\text { Trabajos incompatibles con estado Estilo de vida saludable } \\
\text { de salud }\end{array}$
\end{tabular}

En la figura 5, es posible observar en cuarto lugar, los aspectos relativos a la formación. Los estudios que han sido cursados aunque no se hayan finalizado. También resulta relevante la identificación de las tareas que la persona debe mejorar y el grado de profesionalización que tiene, desarrolla y ha adquirido al finalizar el itinerario formativo. 
Los aspectos más deficitarios que se desprende de la figura 4 y 5 y que forman parte de los indicadores de la empleabilidad en colectivos vulnerables (como por ejemplo, limitaciones horarias en la búsqueda de empleo) hacen referencia a elementos muy específicos, pero que pueden resultar cruciales en la búsqueda de empleo y el fomento del autoempleo, tal y como sugiere el Tratado de Lisboa.

Los aspectos relativos a la salud no parecen suscitar nuevos elementos. Sin embargo, se señalan el estilo de vida que la persona desarrolla diariamente y la incompatibilidad de tareas en función de sus características físicas.

De la categoría de 'Circunstancias personales' únicamente se ha considerado relevante para la evaluación de la empleabilidad recoger información sobre la salud o la dependencia de los familiares con los que convive el trabajador y sobre un aspecto relativo a la movilidad geográfica y flexibilidad laboral, el conocimiento del territorio físico. La movilidad geográfica depende del conocimiento del lugar en donde se vive y se trabaja. Parece que dicho conocimiento no es extensible a toda la población y condiciona la empleabilidad.

\section{Figura 2.}

Porcentaje de aparición de los elementos de las tres dimensiones de la empleabilidad en los instrumentos utilizados por las EIs

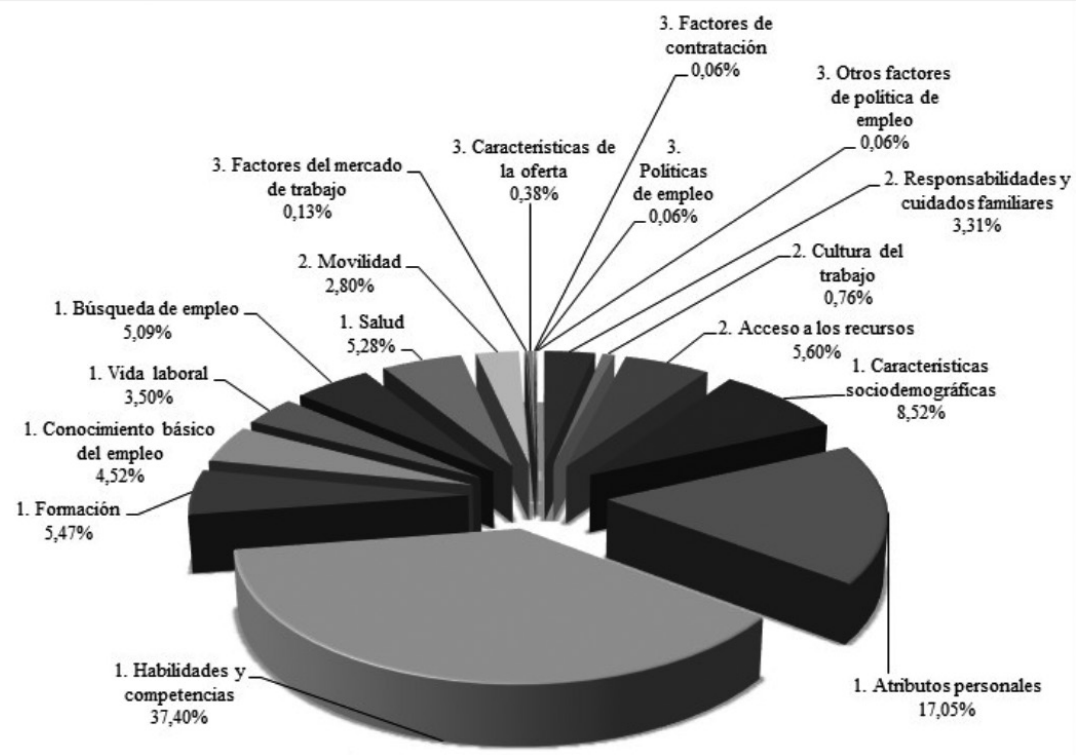

1. Factores individuales

2. Circunstancias personales

3. Factores externos 
Una vez ampliado el IPAIE se llevó a cabo un análisis de contenido de los documentos que miden la empleabilidad en las EI españolas. La figura 2 presenta el porcentaje de los indicadores que miden empleabilidad. Los resultados obtenidos muestran que no hay acuerdo en los agentes sociales vinculados al empleo sobre los ítems concretos de la empleabilidad. Sin embargo, hemos encontrado que, en su mayor parte, la empleabilidad se vincula a factores individuales $(89.70 \%)$, un pequeño porcentaje a circunstancia personal $(9.66 \%)$ y apenas se tienen en cuenta los factores externos $(0,64 \%)$.

Figura 3.

\section{Indicadores básicos de empleabilidad que forman la representación social}

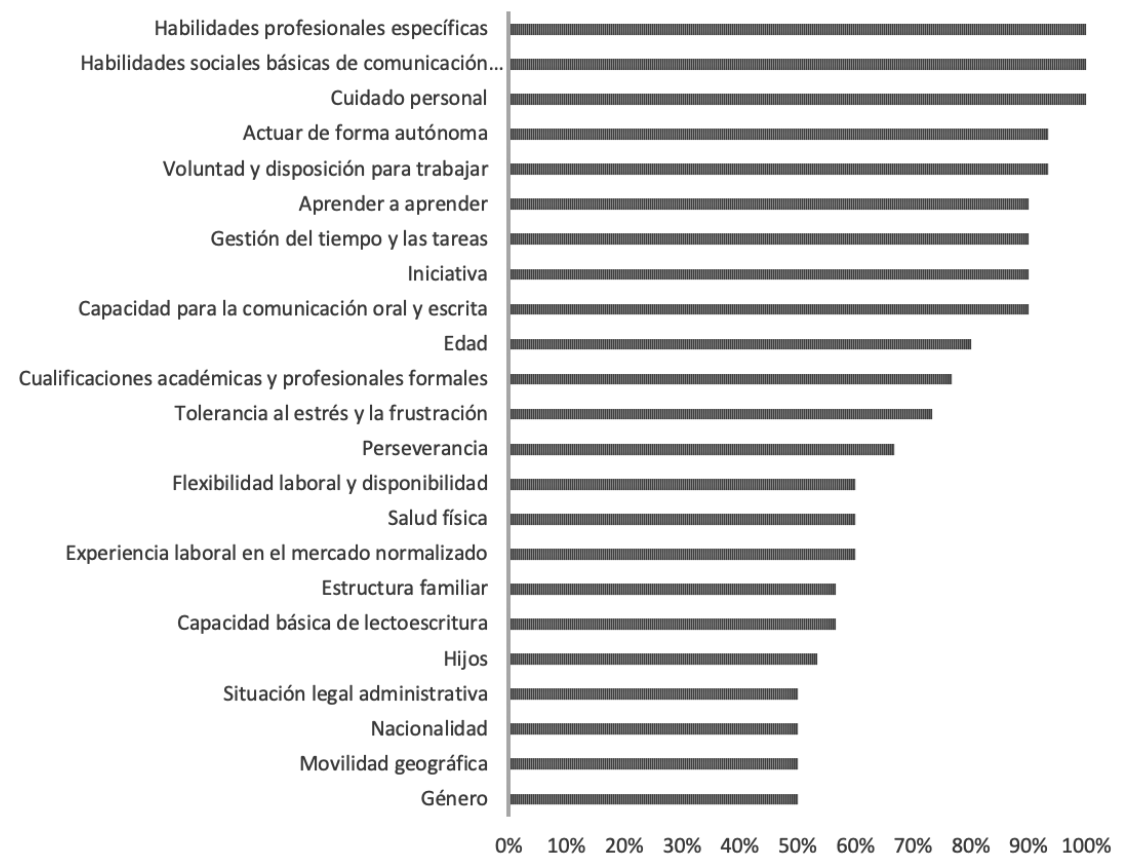

Entre el conjunto de indicadores, y con el fin de conocer la importancia de cada uno de ellos para la medida de la empleabilidad, se llevó a cabo otro análisis descriptivo sobre su uso en la evaluación de la empleabilidad; los resultados se presentan en la figura 3. En ella se muestra que la empleabilidad atiende al cuidado personal, la comunicación interpersonal y las habilidades profesionales específicas junto con la autonomía y la motivación laboral intrínseca. También resultan relevantes aspectos como la edad, las cualificaciones académicas y profesionales y la tolerancia al estrés. 
Menos relevantes en la evaluación de la empleabilidad (50\%) son la perseverancia, la experiencia laboral en el mercado normalizado, la salud, la flexibilidad laboral y la disponibilidad junto con características sociodemográficas (género, nacionalidad y situación legal).

Las circunstancias personales que rodean a la persona no tienen tanta importancia en la evaluación de la empleabildiad. Las más destacadas son las responsabilidades y cuidados familiares; en concreto, la estructura familiar y las cargas familiares en materia de hijos.

La empleabilidad se concibe como una competencia de la persona que incluye atributos personales y que está influida, aunque mínimamente, por las circunstancias personales que la envuelven. El cuidado personal, la comunicación interpersonal, las habilidades profesionales específicas y básicas junto con la autonomía, la voluntad y disposición, la iniciativa, la gestión del tiempo las tareas y el aprendizaje (aprender a aprender y formación previa) y la edad conforman su núcleo central (del $75 \mathrm{al}$ $100 \%)$. Ahora bien, también resultan relevantes la tolerancia al estrés y a la frustración, la perseverancia, la flexibilidad laboral y disponibilidad horaria, la salud y su experiencia laboral.

\section{Discusión}

La empleabilidad es uno de los constructos más usados y también más difusos del ámbito de las Ciencias Sociales (Rozalía, Blaga, y Matis, 2019). Por ello, este trabajo tiene como objetivo el análisis de los indicadores de la empleabilidad desde una perspectiva bioecológica (Llinares-Insa et al, 2016). Para ello se ha tenido en cuenta el análisis de los elementos que evalúan las EI en los itinerarios de inserción de los PTI. Los resultados muestran que la empleabilidad queda integrada por, sobre todo, habilidades (p.e. habilidades sociales específicas y básicas de comunicación, autonomía, gestión del tiempo y las tareas, aprender a aprender) y atributos personales (p.e. voluntad y disposición para trabajar, tolerancia al estrés y la frustración), aunque también se incluyen otras variables importantes (p.e., edad, hijos, nacionalidad, etc.). Destaca, pues, la responsabilidad de la persona en el desarrollo de su carrera laboral tal y como lo indica Fugate, Kinicki y Ashforth (2004) o Salognon (2007). Esta visión requiere que el trabajador desarrolle las competencias relevantes en el mundo laboral y que se sienta responsable de su carrera laboral.

Es el trabajador el que ha de comprometerse por voluntad propia a planificar su futuro profesional y tener una conducta proactiva cuando tenga que buscar oportunidades de trabajo. Así pues, esta perspectiva incide en la responsabilidad individual 
del desarrollo de carrera y permite generar programas para su mejora en todas las instancias sociales vinculadas al empleo y a la formación. Sin embargo, también actúa como elemento legitimador del contexto social y laboral actual. Esto, a su vez, limita la responsabilidad al resto de agentes sociales (De Lara y Andrade, 2008) y no analiza el fenómeno del empleo desde una perspectiva global y holística.

La mejora de la empleabilidad no siempre resulta fácil debido a la competitividad y los recursos disponibles, así como el nivel socioeconómico. Por ello, el aprendizaje y desarrollo de estas competencias debe ir adquiriéndose durante la formación en la empresa y deben perfeccionarse a lo largo de la vida laboral (Vega, Bello y García, 2018). Junto a ello, también se pone de manifiesto que la empleabilidad no depende únicamente de la responsabilidad del individuo, sino también de las necesidades del mercado, las preferencias del empresario, las características del puesto o las circunstancias personales del trabajador, e integra las perspectivas de la oferta y la demanda y la visión desde el mercado de trabajo (Bauman, 2005; Beck y Beck-Genshein, 2002). Por tanto, la empleabilidad es un concepto complejo, que debe incluir desde aspectos macro (estructuras sociales, políticas e ideológicas) a micro (factores individuales y contextos sociales inmediatos) desde los cuales se abordan problemáticas de desempleo y marginalización (Llinares et al., 2016). En este sentido, los datos obtenidos muestran que el núcleo central de la empleabilidad está formado por variables individuales pero que algunas circunstancias personales también la condicionan.

Por tanto, en el presente trabajo, y ante la variedad de aproximaciones al término empleabilidad, hemos podido identificar los indicadores que forman parte del núcleo central de la empleabilidad. Éstos permiten su medida y fomento a todas las instancias políticas, sociales y educativas que están vinculadas a la formación y al empleo. Resulta, pues, una herramienta práctica para la acción educativa y social. También hacemos con ello una aportación teórica ya que quedan identificados los indicadores centrales de la empleabilidad tomando como base el modelo bioecológico de la empleabilidad (Llinares-Insa et al, 2016) y el análisis de la realidad española. Por ultimo, queremos hacer una llamada a la investigación para seguir profundizando en el estudio de los elementos de la empleabilidad que doten a las personas de un desarrollo de carrera integral en un mundo globalizado. De este modo, la empleabilidad se convierte en un instrumento de análisis e intervención, pero también de reflexión. 


\section{Referencias bibliográficas}

ALPEK, L. \& TÉSITS, R. (2019): “Measuring Regional Differences in Employability in Hungary", Applied Spatial Analysis and Policy, 13, 329-347.

DOI: $10.1007 / s 12061-019-09306-6$

AMIGOT, P. \& MARTÍNEZ, L. (2013): "Gestión por competencias, modelo empresarial y sus efectos subjetivos. Una mirada desde la psicología social crítica”, Universitas Psychologica, 12(4), 1073-1084.

ANG, M.C.H. (2015): "Graduate employability awareness: a gendered perspective", Procedia - Social and Behavioral Sciences 211, 192-198.

DOI: $10.1016 /$ j.sbspro.2015.11.083

BALDIVIESO, M.S. (2002): Indicadores para la autoevaluación de instituciones de formación profesional inicial en el marco de la nueva relación educación trabajo (Tesis Doctoral, Universidad Autónoma de Barcelona, Barcelona). Disponible en: https://www.tdx.cat/bitstream/handle/10803/5010/msbh1de6.pdf?sequence $=1 \&$ isAllowed $=y$

BAUMAN, Z. (2005): Legisladores e intérpretes. Sobre la modernidad, la postmodernidad y los intelectuales, Argentina: Universidad Nacional de Quilmes.

BECK, U. \& BECK-GENSHEIN, E. (2002): Individualization, Londres: Sage.

BERNAD, J.C. \& MARTÍNEZ, I. (2011): "Cambios en el contexto de las políticas sociolaborales en el marco del desmontaje de la sociedad salarial”. En Trabajo, empleabilidad y vulnerabilidad social: condicionantes y potencialidades de la integración a través de las Empresas de Inserción Social (CÓRDOBA, A.I. \& MARTÍNEZ, I.), Valencia: SPUV.

BERNSTROM, V.H., DRANGE, I. \& MARMELUND, S.E. (2019): "Employability as an alternative to job security", Personnel Review, 48 (1), 234-248.

DOI: 10.1108/PR-09-2017-0279

BRONFENBRENNER, U. \& MORRIS, P. (1998): "The ecology of developmental process". En Handbook of child psychology: Vol.1. Theoretical models of human development (DAMON, W. (Series Ed.) \& LERNER, R.M. (Vol. Ed.)), New York: Wiley, $5^{\text {th }}$ ed., pp. 993-1028.

BRONFENBRENNER, U. \& MORRIS, P. (2006): "The bioecological model of human development”. En Handbook of child psychology: Vol.1. Theoretical models of human development (DAMON, W. \& LERNER, R.M. (Series Eds.) \& R.M. Lerner (Vol.Ed.)), New York: Wiley, 6 ${ }^{\text {th }}$ ed., pp. 793-828. 
BRUNNER, J.J. (2000): Competencias en empleabilidad. Revisión bibliográfica, Disponible en http://www.geocities.com/brunner_cl/empleab.html

CAMPOS (2003): "Implicaciones económicas del concepto de empleabilidad", Aportes. Revista de la Facultad de Economia-BUAP, 23, 101-111.

CHACÓN SÁNCHEZ, N.V., CASAS MATEUS, D.E. \& ALVARADO NIETO, L.D. (2020): "Behavior of employability indicators in university graduates", $R e-$ vista Ingeniería Solidaria, 16(2).

DOI: https://doi.org/10.16925/2357-6014.2020.02.03

CÓRDOBA, A.I., LLINARES, L.I. \& ZACARÉS, J.J. (2013): “Employability Assessment in Vocational Education and Transition to the Workplace". En Transitions in Vocational Education, Research in Vocational Education (Eds. SEIFRIED, J. \& WUTTKE, E.), Opladen, Berlin, Farmington Hills: Budrich publisher, Vol. 2, pp. 181-200.

DE CUYPER, N., MAUNO, S., KINNUNEN, U. \& MAKIKANGAS, A. (2011): "The role of job resources in the relation between perceived employability and turnover intention: A prospective two-sample study", Journal of Vocational Behavior, 78(2), 253-263.

DE GRIP, A., VAN LOO, J. \& SANDERS, J. (2004): “The Industry Employability Index: Taking Account of Supply and Demand Characteristics", International Labour Review, 143(3), 211-233.

DE LARA, K.C. \& ANDRADE, F. (2008): "Empregabilidade: Construçao de uma escala", PsicoUSF, 13(2), 189-201.

DRANGE, I., BERNSTRØM, V.H. \& MAMELUND, S.E. (2018): "Are you moving up, or falling out? An inquiry of skill-based variation in self-perceived employability among Norwegian employees", Work, Employment and Society, 32(2), 387-406. DOI: 10.1177/0950017017749720.

EBUENYI, I.D., GUXENS, M., OMBATI, E., BUNDERS-AELEN, J.F.G. \& REGEER, B.J. (2019): "Employability of Persons With Mental Disability: Understanding Lived Experiences in Kenya", Front. Psychiatry, 10, 539.

DOI: $10.3389 /$ fpsyt.2019.00539

FAEDEI \& AERES (2014): El acompañamiento en las empresas de inserción, Madrid: AERES- FAEDEI.

FAHIMIRAD, M., NAIR, P.K., KOTAMJANI, S.S., MAHDINEZHAD, M. \& FENG, J.B. (2019): "Integration and development of Employability Skills into Malaysian Higher Education Context: Review of the Literature", International Journal of Higher Education, 8(6), 26-35. DOI: 10.5430/ijhe.v8n6p26

FINN, D. (2000): "From full employment to full employability: a new deal for Britain's unemployed?”, International Journal of Manpower, 21, 384-399. 
FORMICHELLA, M.M. \& LONDON, S. (2005): "Reflexiones acerca de la noción de empleabilidad", Anales, Trabajo presentado en las reuniones de la AAEP, Buenos Aires Argentina.

Disponible en www.aaep.org.ar/espa/anales/works05/formichella_london.pdf

FORRIER, A. \& SELS, L. (2003): “Temporary employment and employability: training opportunities and efforts of temporary and permanent employees in Belgium", Work Employment and Society, 17(4), 641-666.

FUGATE, M. \& KINICKI, A.J. (2008): "A dispositional approach to employability: Development of a measure and test of implications for employee reactions to organizational change", Journal of Occupational and Organizational Psychology, 81(3), 503-527.

FUGATE, M., KINICKI, A.J. \& ASHFORTH, B.E. (2004): "Employability: A psycho-social construct, its dimensions, and applications", Journal of Vocational Behavior, 65(1), 14-38.

GABOR, M., BLAGA, P. \& MASTIS, C. (2019): "Supporting employability by a skills assessment innovativa tool-Sustainable transnational insights from employers", Sustainability, 11, 3360, 1-19. DOI: 10.3390/su11123360

GARCÍA, M.I. \& IBÁÑEZ, M. (2006): “Competencias para el empleo, demandas de las empresas y medición de los ajustes”, Revista internacional de Sociología, 43, 139-168.

HAZELZET, E., PICCO, E., HOUKES, I., BOSMA, H \& DE RIJK, A. (2019): "Effectiveness of Interventions to Promote Sustainable Employability: A Systematic Review”, Int. J. Environ. Res. Public Health, 16. DOI:10.3390/ijerph16111985 HOGAN, R., CAHMORRO-PREMUZIC, T. \& KAISER, R.B. (2013): "Employability and career success: bridging the gap between theory and reality", Industrial and Organizational Psychology, 6, 3-16.

JOL, M.M., STOFFERS, B., VAN DER HEIJDEN, I.J.M. \& JACOBS, E.A.G.M. (2020): "Employability and innovative work behaviour in small and medium-sized enterprises", The International Journal of Human Resource Management, 31(11), 1439-1466. doi: 10.1080/09585192.2017.1407953

LINDSAY, C. \& SERRANO, M.A. (2009): "New perspectives on employability and labour market policy: reflecting on key issues", Environment and Planning C: Government and Policy, 6(27), 951-957.

LLINARES, L., CÓRDOBA, A.I. \& ZACARÉS, J.J. (2012): "La medida de la empleabilidad en las empresas de inserción en el País Vasco: de la exclusión a la inserción socio-laboral", Zerbitzuan, 51, 83-94. 
LLINARES, L.I., CÓRDOBA, A.I. \& ZACARÉS, J.J. (2011): "La medida de la empleabilidad desde las Empresas de Inserción Social”. En Trabajo, empleabilidad y vulnerabilidad social: condicionantes y potencialidades de la integración a través de las Empresas de Inserción Social (CÓRDOBA, A.I. \& MARTÍNEZ, I.), Valencia, SPUV, pp. 131-181.

LLINARES, L.I., ZACARÉS, J.J. \& CÓRDOBA, A.I. (2016): "Discussing employability: current perspectives and key elements from a bioecological model", Employee Relations, 38(6), 961-974.

MARHUENDA FLUIXÁ, F. (2018): “Aprendizaje en el puesto de trabajo: investigar el desarrollo personal y la adquisición de competencias en empresas de inserción”, Educar, 54(2), 491-509.

MARTÍNEZ RUEDA, N., GALARRETA, J. \& ARÓSTEGUI, I. (2018): "Empleabilidad y empresas de inserción: modelo de prestaciones y apoyos", Zerbizuan, 67, 79-93. DOI: 10.5569/1134-7147.67.06

MCDONALD, P., GRANT-SMITH, D., MOORE, K. \& MARSTON, G. (2020): "Navigating employability from the bottom up", Journal of Youth Studies, 23(4), 447-464. DOI: 10.1080/13676261.2019.1620925

MCQUAID, R.W. \& LINDSAY, C. (2005): "The concept of employability", Urban Studies, 42(2), 197-219.

MOHD, HUSAIN, Y., MUSTAPHA, R., MALIK, S.A., MOHAMAD, S., RASUL, M.S. \& MOKHTAR, S.B. (2013): Employability Skills, Co-curriculum Management, Peer Interaction and Contextual Teachingand Learning in Technical Institutions.

MORELAND, N. (2006): "Entrepreneurship and Higher Education: An Employability Perspective", Learning y Employability Series One, Nueva York: UK, ESECT. NAUTA, A., A. VAN VIANEN, B. VAN DER HEIJDEN, K. VAN DAM, \& M. WILLEMSEN (2009): "Understanding the Factors That Promote Employability Orientation: The Impact of Employability Culture, Career Satisfaction, and Role Breadth Selfefficacy", Journal of Occupational \& Organizational Psychology, 82 (2): 233-251.

OLSON, D.A. \& SHULZ, K.S. (2013): "Employability and career success: The need for comprehensive definitios of career success", Industrial and Organizational Psychology, 6, 17-38.

PÉREZ, C. (2003): "El Discurso de la Empleabilidad y las Funciones del Sistema Educativo. Repercusiones para la Sociología de la Educación”, X Conferencia de Sociología. Disponible en http://www.uv.es/ -jbeltran/ase/textos/perezsanchez.pdf. 
PORTA, L. \& SILVA, M. (2003): "La investigación cualitativa: El Análisis de Contenido en la investigación educativa", Anuario Digital de Investigación Educati$v a, 14,1-18$.

RECIO, A. \& BANYULS, J. (2004): "Employment without technology? The paradox of Spanish Labour Market”, Economia et Lavoro, 38(1), 115-137.

RENTERÍA, E. \& MALVEZZI, S. (2008): "Empleabilidad, cambios y exigencias psicosociales en el trabajo", Universitas Psychologica, 2(2), 319-334.

ROZALÍA, M., BLAGA, P. \& MATIS, C. (2019): "Supporting employability by a skills assessment innovative tool-Sustainable transnational insignhts from employers", Sustainability, 11, 3360, 1-18. DOI: 10.3390/su11123360

SALOGNON, M. (2007): "Re-Orienting Compagnies's Hiring Behaviour: An Innovative 'Back-To-Work' Method in France", Work, Employment and Society, 21(4), 713-730.

SANTOS, B. (2003): Crítica de la razón indolente. Contra el desperdicio de la experiencia, Bilbao: Desclée de Brouwer.

SERRANO, M.A. (1999): "The concept of employability: A critical assessment to fight against youth unemployment", European Trade Union Yearbook 01/01/2000, Brussels, ETUI, 253-262.

SHULTZ, K.S. \& OLSON, D.A. (2013): "The changing nature of work and retirement”. En The Oxford handbook of retirement (Ed. WANG, M.), New York, NY: Oxford University Press, pp. 543-558.

STORME, E., DE LANNOY, A., LEIBBRANDT, M., DE BOECK, F., MUDIRIZA, G. (2019): Developing a Multidimensional Youth Employability Index to unpack vulnerabilities in the lived realities of youth in Post-apartheid South Africa, Cape Town: SALDRU, UCT.

TERZAROLI, C. \& OYEKUNLE, Y. (2019): "Career service as a measure to support employability: A comparison between the university of Florence and the University of Lagos", Andragoška spoznanja/Studies in Adult Education and Learning, 25(1), 89-110.

THIJSSEN, J.G.L., VAN DER HEIJDEN, B.I. J.M. \& ROCCO, T.S. (2008): "Toward the employability link model: Current employment transition to future employment perspectives", Human Resource Development Review, 7(2), 165-183. UNESCO (2015): Declaración de Incheon y Marco de Acción ODS 4-Educación 2030. Disponible en http://www.unesco.org/new/es/education/themes/leading-the-international-agenda/education-for-all/sdg4-education-2030

VEGA, L.E.S., BELLO, E.A. \& GARCÍA, L.F. (2018): “Trayectorias laborales y competencias de empleabilidad de jóvenes nacionales e inmigrantes en riesgo de exclusión social", Revista Complutense de Educación, 29(2), 355-369. 
VENTURA, J. (2005): El prácticum en los estudios pedagógicos y la inserción laboral. Nuevos enfoques ante el reto europeo. Tesis doctoral, Universitat de Barcelona, Barcelona, España.

WILLIAMS, S., DODD, L.J., STEELE, C. \& RANDALL, R. (2016): "A systematic review of current understandings of employability", Journal of Education and Work, 29 (8), 877-901. DOI: 10.1080/13639080.2015.1102210 